\title{
Georeferenced Status of Cobalt in Soils of Yavatmal District of Maharashtra
}

\author{
Kabir Debbarma, R. N. Katkar, S.R. Lakhe \\ Department of Soil Science, Dr. PDKV, Akola-444104, India
}

\begin{abstract}
The present study entitled "Georeferenced Status of cobalt in soils ofYavatmal district of Maharashtra" was conducted during 2012-13 at Department of Soil Science and Agricultural Chemistry, Dr. PDKV, Akola with objective to assess the cobaltstatus in soils of Yavatmal district and to study relationship of cobalt with soil properties and preparation of GIS based soil fertility maps of micronutrients. In the Yavatmal district, the cobalt ranged $0.02-1.80 \mathrm{mg} \mathrm{kg}^{-1}$. Based on critical limit of cobalt $\left(0.25 \mathrm{mg} \mathrm{k} \mathrm{k}^{-1}\right)$ the deficiency was noticed 61.97 percent. The nutrient indices of DTPA-Co were 1.67 respectively. DTPA-cobalt showed negative and significant correlation with soil $\mathrm{pH}(-0.142 * *)$. The calcium carbonate content of soil showed negative and significant correlation with DPTA-cobalt $\left(r=-0.104^{*}\right)$. It could be concluded that, in Yavatmal district the soils were found deficient in cobalt (61.97\%).
\end{abstract}

Keywords: Cobalt, Diethylene triamine penta acetic acid, Geographical information system, Nutrient indices, Thematic map

\section{Introduction}

Soil is the mother for supporting and nourishing all life on the earth. It is most precious natural resource which requires several years to develop a inch of soil. Its proper use generally determines the capability of life support system and socio-economic development of any nation. Being an important component of our geosphere-biosphere system, soil provide food, fibre, fodder and fuel wood for various of basic human needs, their contribution on vegetation and control of water and nutrient cycles.

Soils of Maharashtra state are categorized as poor to moderate in fertility and they are widely in genetic, morphological, physical, chemical and biological characteristics. The nature and behaviour in soil of the essential and beneficial plant nutrients viz., $\mathrm{Zn}, \mathrm{Fe}, \mathrm{Mn}, \mathrm{Cu}, \mathrm{B}, \mathrm{Mo}$ and $\mathrm{Co}$ traditionally classed as micronutrients needed in small quantity by plants and their reserves are limited. Micronutrient has emerged to show prominence in Indian agriculture only after their deficiency. These are depleted due to continuous cropping, soil erosion, leaching and with the use of high analysis fertilizers in intensive cropping system with less use of organic manures.

In recent years, the cobalt content of soils and plants has become important because of the relationship between animal nutrition and the cobalt content of plants. As a constituent of vitamin $\mathrm{B}_{12}$ in nodules, cobalt is essential to the nutrition of some and possibly all animals. Plants accumulate cobalt from the soil and then constitute the primary source of these elements for ruminant.

\section{Material And Methods}

Georeferenced surface $(0-20 \mathrm{~cm})$ soil samples representing different soils were collected tehsilwise soil samples collected during 2011-12 under the "Nutrient Project on Management of Soil Health and Fertility for GPS and GIS Based Model Soil Fertility Maps" were used for the present investigation. The latitude, longitude and altitude of sampling sites in the study area were recorded with the help of Geographical Positioning System (GPS).The soil samples were collected from the farmer's fields of dominant crops. Soil $\mathrm{pH}$ was determined with the help of pH meter in 1:2.5 soils: water suspension as described by Jackson (1973). Organic carbon was determined by Walkely and Black wet-oxidation method as given by Nelson and Sommer (1982). Calcium carbonate was determined by rapid titration method as given by Piper (1966).DTPA- Cobalt was determined by using $0.005 \mathrm{M}$ DTPA adjusted at $\mathrm{pH}$ 7.3. The soil samples were shaked in temperature controlled incubator for $2 \mathrm{hrs}$ with constant temperature $25^{\circ} \mathrm{C}$ and $120 \mathrm{rpm}$. The suspensions were filtered through Whatman No. 42 filter paper. The aliquot was used for analysis. The concentration of Co was recorded on Atomic Absorption Spectrophotometer (AAS) as per procedure given by Lindsay and Norvell (1978). The nutrient indices were calculated by using formula given by Parkar (1951).The DTPA- Cobalt status areas were delineated into low, medium and high using latitude, longitude and data of each sampling sites and thematic maps were prepared using geographical information system (GIS).The correlation of DTPA- Cobalt with $\mathrm{pH}$, organic carbon and calcium carbonate were determined as described by Panse and Sukhatme (1985). 


\section{Results And Discussion}

The data pertaining to the soil $\mathrm{pH}$ in Yavatmal district regarding the samples selected Co analysis is reported in Table 1. The $\mathrm{pH}$ ranges from 6.88 to 8.56 indicating neautral to moderately alkaline in reaction. The highest $\mathrm{pH}$ was recorded in Darva tehsil (8.56) and lowest value in Yavatmal tehsil (6.88). The calcium carbonate of soils ranged from the 1.00 to 15.00 per cent which indicated that soils selected for cobalt analysis were slightly-calcareous, moderately to highly calcareous in nature. The organic carbon content varied from 1.35 to $9.73 \mathrm{~g} \mathrm{~kg}^{-1}$. The organic carbon variation could be noticed in every tehsils with highest content in Zarijamni $\left(9.73 \mathrm{~g} \mathrm{~kg}^{-1}\right)$ followed by Digrus $\left(8.97 \mathrm{~g} \mathrm{~kg}^{-1}\right)$ tehsil and lowest in case of Ner and Umarkhed tehsils $\left(1.35 \mathrm{~g} \mathrm{~kg}^{-1}\right)$.

The DTPA cobalt was found in Yavatmal district vary from 0.02 to $1.80 \mathrm{mg} \mathrm{kg}^{-11}$. The DTPA cobalt in Yavatmal district was found to be in low (61.97\%), medium (8.07\%) and high (29.94\%) category (Table 2 and Fig.2). The highest deficiency of cobalt was found in Babulgaon (100\%) tehsil, followed by Darva (91.66\%) tehsil of Yavatmal district while lowest deficiency was found in Umarkhed tehsil (25.00\%). The nutrient index of Umarkhed tehsil was found (2.33) followed by Ghatanji tehsil (2.29) of Yavatmal district. The lowest nutrient index was found in Babulgaon tehsil (1.00) of Yavatmal district. Randhawa and Kanwar (1964) reported that total cobalt in Punjab soils varies from 4.4 to $32.5 \mathrm{ppm}$, with an average of $20.2 \mathrm{ppm}$. The negative and significant correlation of cobalt with soil $\mathrm{pH}(\mathrm{r}=-0.142 * *)$ (Table 3$)$ and negatively significantly correlation with $\mathrm{CaCO}_{3}(\mathrm{r}=-0.104 *)$ was noticed. Bansal et al $(1991)$ reported that the available cobalt deficiency increased with the decrease in soil $\mathrm{pH}$ and $\mathrm{CaCO}_{3}$ content.

Table 1. Status of $\mathrm{pH}, \mathrm{CaCO}_{3}$ and organic carbon in soils of Yavatmal district

\begin{tabular}{|c|c|c|c|c|}
\hline $\begin{array}{l}\text { Sr. } \\
\text { No. }\end{array}$ & Tehsils & $\begin{array}{c}\text { Soil pH (1:2.5) } \\
\text { range }\end{array}$ & $\begin{array}{c}\mathrm{CaCO}_{3} \\
\text { range (\%) }\end{array}$ & $\begin{array}{c}\text { Organic carbon } \\
\text { range }\left(\mathrm{g} \mathrm{kg}^{-1}\right)\end{array}$ \\
\hline 1 & $\begin{array}{l}\text { Ner } \\
(24)\end{array}$ & 7.04-8.24 & $1.50-11.60$ & $1.50-6.83$ \\
\hline 2 & $\begin{array}{l}\text { Babulgaon } \\
\text { (24) }\end{array}$ & $7.12-8.04$ & $1.75-15.00$ & $1.50-7.87$ \\
\hline 3 & $\begin{array}{c}\text { Kalamb } \\
\text { (24) }\end{array}$ & $7.01-8.05$ & $1.50-11.25$ & $2.93-8.80$ \\
\hline 4 & $\begin{array}{l}\text { Yavatmal } \\
\text { (24) }\end{array}$ & $6.88-8.26$ & $3.25-12.01$ & $1.82-8.55$ \\
\hline 5 & $\begin{array}{c}\text { Darva } \\
(24)\end{array}$ & $7.20-8.56$ & $5.87-12.12$ & $2.05-8.34$ \\
\hline 6 & $\begin{array}{l}\text { Digrus } \\
(24)\end{array}$ & $7.65-8.46$ & $7.50-12.37$ & $2.63-8.97$ \\
\hline 7 & $\begin{array}{l}\text { Pusad } \\
\text { (24) }\end{array}$ & $7.14-7.85$ & $1.37-12.50$ & $2.10-8.69$ \\
\hline 8 & $\begin{array}{l}\text { Umarkhed } \\
\text { (24) }\end{array}$ & $7.20-7.95$ & $1.70-12.00$ & $1.35-7.85$ \\
\hline 9 & $\begin{array}{l}\text { Mahagaon } \\
\text { (24) }\end{array}$ & $7.09-8.39$ & $4.25-11.75$ & $2.25-7.35$ \\
\hline 10 & $\begin{array}{l}\text { Arni } \\
\text { (24) }\end{array}$ & 7.04-8.39 & $1.62-12.12$ & $2.23-7.13$ \\
\hline 11 & $\begin{array}{c}\text { Ghatanji } \\
(24)\end{array}$ & $7.06-8.25$ & $1.25-12.00$ & $2.57-8.00$ \\
\hline 12 & $\begin{array}{c}\text { Pandharkawada } \\
\text { (24) }\end{array}$ & $7.18-8.37$ & $2.37-12.87$ & $3.73-8.27$ \\
\hline 13 & $\begin{array}{c}\text { Ralegaon } \\
\text { (24) }\end{array}$ & $7.18-8.38$ & $1.62-12.12$ & $3.47-8.13$ \\
\hline 14 & $\begin{array}{l}\text { Maregaon } \\
\text { (24) }\end{array}$ & $7.21-8.10$ & $2.00-10.87$ & $2.53-7.73$ \\
\hline 15 & $\begin{array}{l}\text { ZariJamni } \\
\text { (24) }\end{array}$ & $6.91-7.67$ & $1.00-12.25$ & $2.13-9.73$ \\
\hline \multirow[t]{2}{*}{16} & $\begin{array}{l}\text { Wani } \\
(24)\end{array}$ & $7.02-8.30$ & $1.37-12.25$ & $2.53-8.40$ \\
\hline & $\begin{array}{c}\text { Yavatmal district } \\
(\mathbf{3 8 4})\end{array}$ & $6.88-8.56$ & $1.00-15.00$ & $1.35-9.73$ \\
\hline
\end{tabular}

(Values in parenthesis indicate number of samples) 
Table 2. Status of DTPA-cobalt $\left(\mathrm{mg} \mathrm{kg}^{-1}\right)$ and nutrient indices in soils of Yavatmal district

\begin{tabular}{|c|c|c|c|c|c|c|}
\hline \multirow{2}{*}{$\begin{array}{l}\text { Sr. } \\
\text { No. }\end{array}$} & \multirow[t]{2}{*}{ Tehsils } & \multirow{2}{*}{$\begin{array}{c}\text { Range } \\
\left(\mathrm{mg} \mathrm{Kg}^{-1}\right)\end{array}$} & \multicolumn{3}{|c|}{ Status of cobalt } & \multirow{2}{*}{$\begin{array}{l}\text { Nutrient } \\
\text { Indices }\end{array}$} \\
\hline & & & $\begin{array}{c}\text { Low } \\
(<0.25)\end{array}$ & $\begin{array}{c}\text { Medium } \\
(\mathbf{0 . 2 5 - 0 . 3 5 )}\end{array}$ & $\begin{array}{l}\text { High } \\
(>\mathbf{0 . 3 5})\end{array}$ & \\
\hline 1 & $\begin{array}{l}\text { Ner } \\
(24)\end{array}$ & $0.03-0.36$ & $\begin{array}{c}23 \\
(95.83)\end{array}$ & $\begin{array}{c}0 \\
(0.00)\end{array}$ & $\begin{array}{c}1 \\
(4.16)\end{array}$ & 1.07 \\
\hline 2 & $\begin{array}{l}\text { Babulgaon } \\
\text { (24) }\end{array}$ & 0.03-0.09 & $\begin{array}{c}24 \\
(100.00)\end{array}$ & $\begin{array}{c}0 \\
(0.00)\end{array}$ & $\begin{array}{c}0 \\
(0.00)\end{array}$ & 1.00 \\
\hline 3 & $\begin{array}{c}\text { Kalamb } \\
\text { (24) }\end{array}$ & $0.03-0.91$ & $\begin{array}{c}17 \\
(70.83)\end{array}$ & $\begin{array}{c}1 \\
(4.16)\end{array}$ & $\begin{array}{c}6 \\
(25.00)\end{array}$ & 1.54 \\
\hline 4 & $\begin{array}{l}\text { Yavatmal } \\
\text { (24) }\end{array}$ & $0.02-0.38$ & $\begin{array}{c}20 \\
(83.3)\end{array}$ & $\begin{array}{c}3 \\
(12.50)\end{array}$ & $\begin{array}{c}1 \\
(4.16)\end{array}$ & 1.20 \\
\hline 5 & $\begin{array}{c}\text { Darva } \\
(24)\end{array}$ & $0.03-0.50$ & $\begin{array}{c}22 \\
(91.66)\end{array}$ & $\begin{array}{c}0 \\
(0.00)\end{array}$ & $\begin{array}{c}2 \\
(8.34)\end{array}$ & 1.16 \\
\hline 6 & $\begin{array}{l}\text { Digrus } \\
\text { (24) }\end{array}$ & $0.06-1.42$ & $\begin{array}{c}16 \\
(66.67)\end{array}$ & $\begin{array}{c}3 \\
(12.50)\end{array}$ & $\begin{array}{c}5 \\
(20.83)\end{array}$ & 1.54 \\
\hline 7 & $\begin{array}{c}\text { Pusad } \\
\text { (24) }\end{array}$ & $0.02-1.80$ & $\begin{array}{c}21 \\
(87.5)\end{array}$ & $\begin{array}{c}0 \\
(0.00)\end{array}$ & $\begin{array}{c}3 \\
(12.50)\end{array}$ & 1.25 \\
\hline 8 & $\begin{array}{l}\text { Umarkhed } \\
\text { (24) }\end{array}$ & $0.07-1.13$ & $\begin{array}{c}6 \\
(25.00)\end{array}$ & $\begin{array}{c}4 \\
(16.67)\end{array}$ & $\begin{array}{c}14 \\
(58.34)\end{array}$ & 2.33 \\
\hline 9 & $\begin{array}{l}\text { Mahagaon } \\
\text { (24) }\end{array}$ & $0.09-1.14$ & $\begin{array}{c}10 \\
(41.67)\end{array}$ & $\begin{array}{c}2 \\
(8.34)\end{array}$ & $\begin{array}{c}12 \\
(50.00)\end{array}$ & 2.08 \\
\hline 10 & $\begin{array}{l}\text { Arni } \\
\text { (24) }\end{array}$ & $0.16-1.49$ & $\begin{array}{c}10 \\
(41.67)\end{array}$ & $\begin{array}{c}6 \\
(25.00)\end{array}$ & $\begin{array}{c}8 \\
(33.34)\end{array}$ & 1.91 \\
\hline 11 & $\begin{array}{c}\text { Ghatanji } \\
\text { (24) }\end{array}$ & $0.03-1.07$ & $\begin{array}{c}8 \\
(33.34)\end{array}$ & $\begin{array}{c}1 \\
(4.16)\end{array}$ & $\begin{array}{c}15 \\
(62.50)\end{array}$ & 2.29 \\
\hline 12 & $\begin{array}{c}\text { Pandharkawada } \\
\text { (24) }\end{array}$ & $0.02-0.86$ & $\begin{array}{c}21 \\
(87.5)\end{array}$ & $\begin{array}{c}0 \\
(0.00)\end{array}$ & $\begin{array}{c}3 \\
(12.50)\end{array}$ & 1.25 \\
\hline 13 & $\begin{array}{l}\text { Ralegaon } \\
\text { (24) }\end{array}$ & $0.08-0.84$ & $\begin{array}{c}13 \\
(54.16)\end{array}$ & $\begin{array}{c}2 \\
(8.34)\end{array}$ & $\begin{array}{c}9 \\
(37.50)\end{array}$ & 1.70 \\
\hline 14 & $\begin{array}{l}\text { Maregaon } \\
\text { (24) }\end{array}$ & $0.15-1.13$ & $\begin{array}{c}8 \\
(33.34)\end{array}$ & $\begin{array}{c}6 \\
(25.00)\end{array}$ & $\begin{array}{c}10 \\
(41.67)\end{array}$ & 2.08 \\
\hline 15 & $\begin{array}{l}\text { Zarijamni } \\
\text { (24) }\end{array}$ & $0.04-1.00$ & $\begin{array}{c}9 \\
(37.50)\end{array}$ & $\begin{array}{c}3 \\
(12.50)\end{array}$ & $\begin{array}{c}12 \\
(50.00)\end{array}$ & 2.12 \\
\hline 16 & $\begin{array}{l}\text { Wani } \\
(24)\end{array}$ & $0.14-1.34$ & $\begin{array}{c}10 \\
(37.50)\end{array}$ & $\begin{array}{c}0 \\
(12.50)\end{array}$ & $\begin{array}{c}14 \\
(58.34)\end{array}$ & 2.16 \\
\hline & $\begin{array}{c}\text { Yavatmal district } \\
(384)\end{array}$ & $0.02-1.80$ & $\begin{array}{c}239 \\
(61.97)\end{array}$ & $\begin{array}{c}31 \\
(\mathbf{8 . 0 7 )}\end{array}$ & $\begin{array}{c}115 \\
(29.94)\end{array}$ & 1.67 \\
\hline
\end{tabular}

(Values in parenthesis are percentage, NI: Low <1.66, Medium 1.66-2.33, High >2.33)

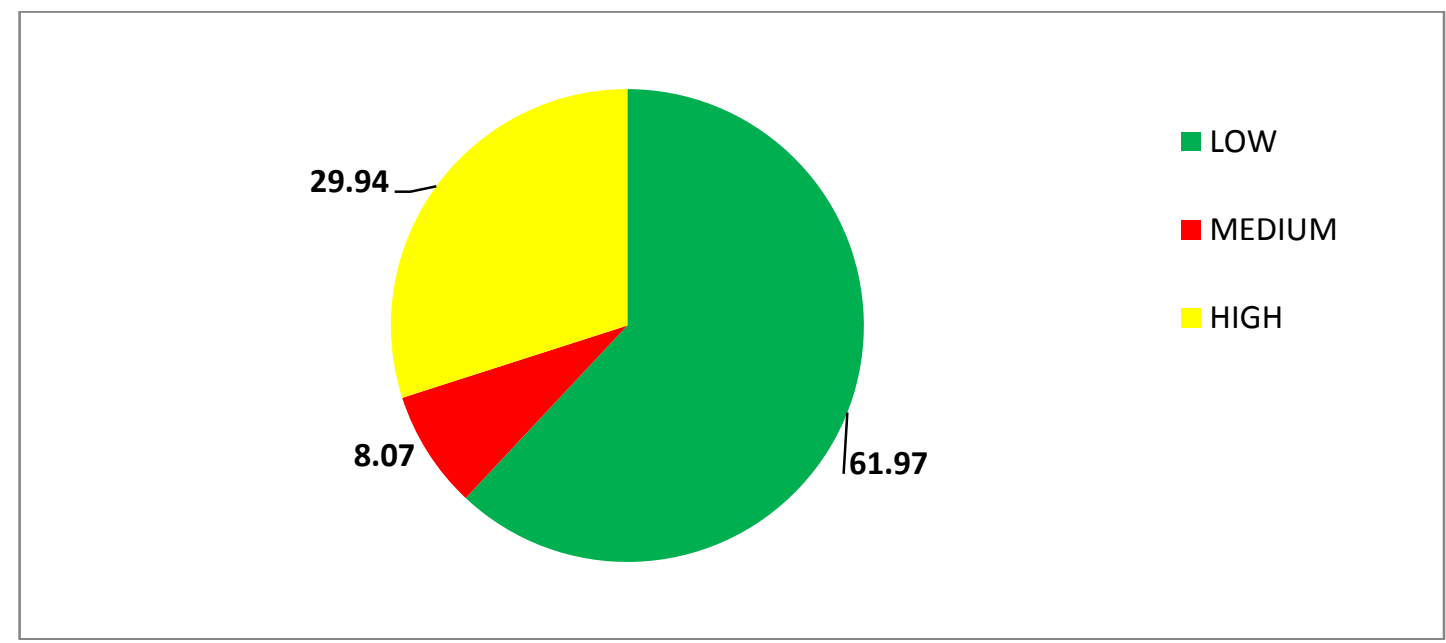

Fig 1. Status of DTPA-Cobalt in Yavatmal districtl 


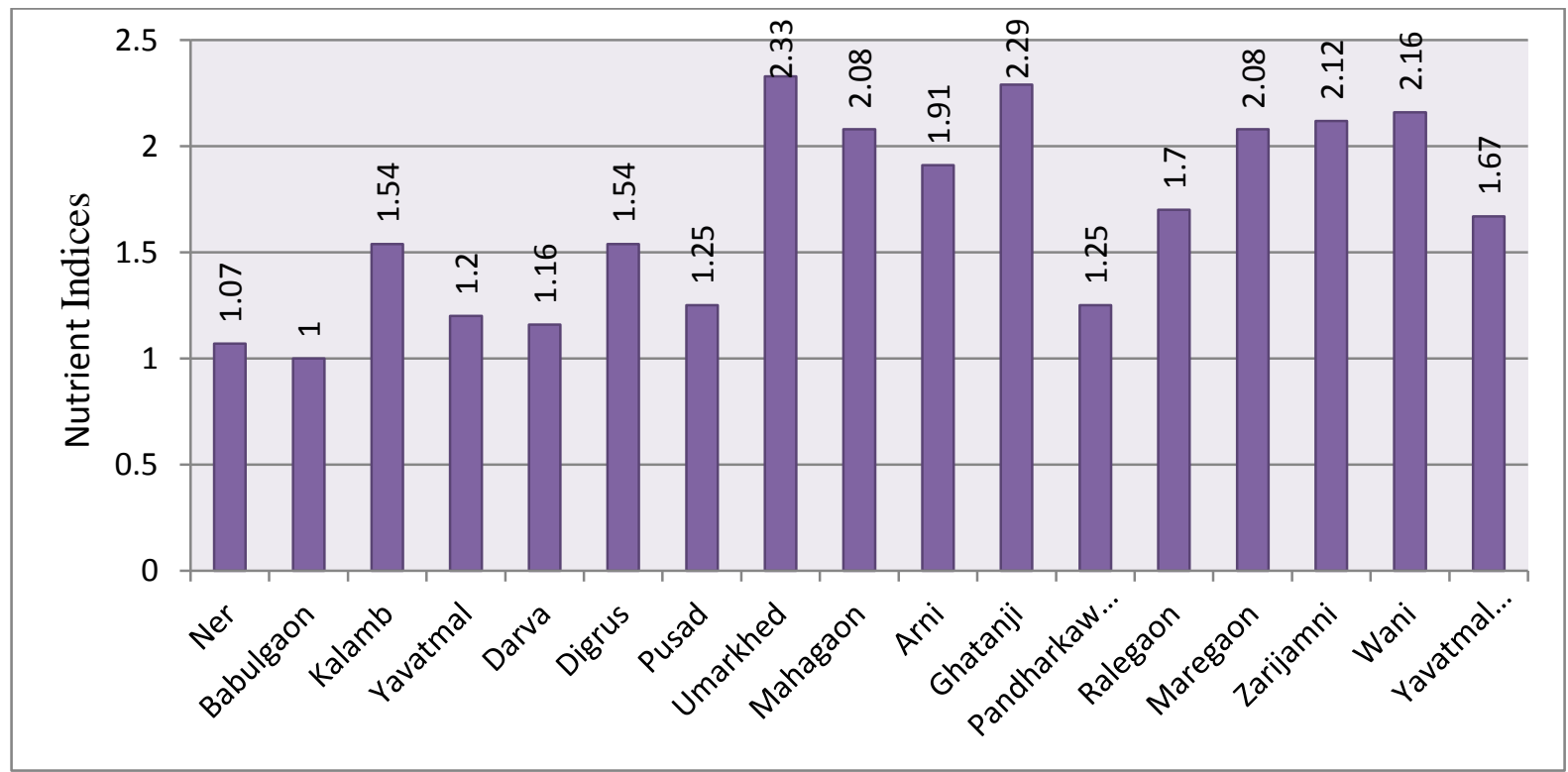

Fig 2.Tehsiwise nutrient indices of cobalt in Yavatmal district

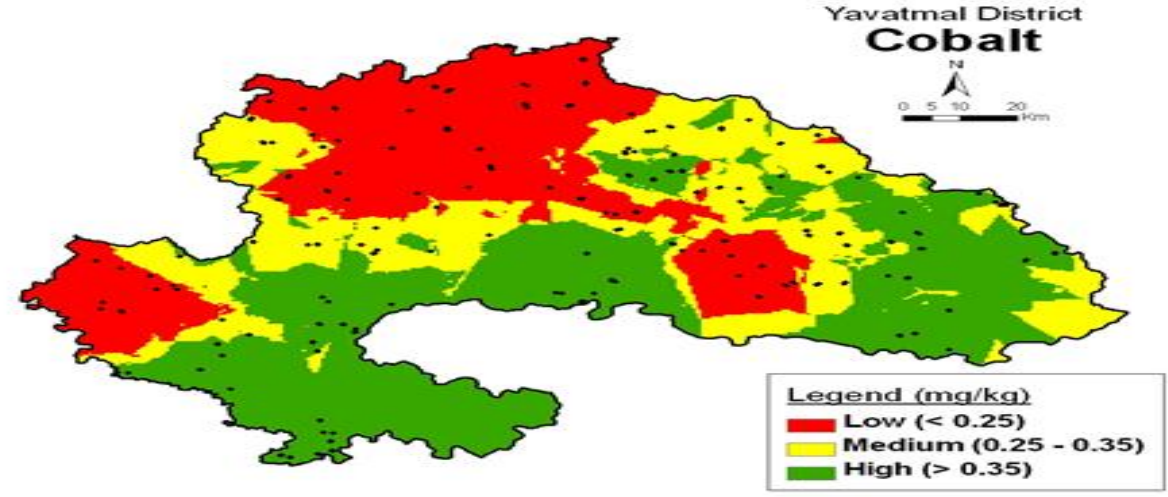

Fig 3. Status of DTPA Cobalt in soils of Yavatmal district

Table 3. The relationship of Cobalt was studied with other soil properties.

\begin{tabular}{c|l|c}
\hline $\begin{array}{c}\text { Sr. } \\
\text { No. }\end{array}$ & Parameters & $\begin{array}{c}\text { Co } \\
(\mathbf{n = 3 8 4})\end{array}$ \\
\hline 1. & $\mathrm{pH}$ & $-0.142^{* *}$ \\
\hline 3. & $\mathrm{CaCO}_{3}$ & $-0.104^{*}$ \\
\hline
\end{tabular}

$*_{-}$Significant at $5 \%$ level

**-Significant at $1 \%$ level

\section{Conclusions}

The soils of Yavatmal district were found to be neutral to alkaline in nature (6.88 to 8.56) with low to medium organic carbon content (1.35-9.73 $\left.\mathrm{g} \mathrm{kg}^{-1}\right)$.In Yavatmal district, the content of cobalt ranged from0.02 to $1.80 \mathrm{mg} \mathrm{kg}^{-1}$, respectively. The deficiency was noticed 61.97 percent.

\section{References}

[1]. Jackson, M. L., 1973. Soil Chemical Analysis (Eds.) Prentice Hall of India Pvt. Ltd., New Delhi. pp 69-182.

[2]. Piper, C. S., 1966. Soil and Plant Analysis, Hans. Pub. Bombay. Asian Ed. pp 368-374.

[3]. Walkely, A and C.A. Black, 1934. The method for determining soil organic matter and proposed modification of chromic acid titration method, Soil Sci. (37): 28-29.

[4]. Lindsay, W.L. and W.A. Norvell, 1978. Development of DTPA soil test for zinc, iron, manganese, copper and cobalt. Soil Sci. Am. J. 42:421-428

[5]. Randhawa, N.S. and J.S. Kanwar, 1964. Zinc, copper and cobalt status of Punjab soil. Agricultural university, Ludhiana: 403-407.

[6]. Bansal, S., V.K. Nayyar and P.N. Takkar, 1991. Availability of B, Co and S in Ustochrepts. J. Indian Soc. Soil Sci. 39: 181-182. 